\title{
Rehabilitation und Doping - Sachverstand ist gefragt
}

\author{
Bei der diesjährigen Hannover Herz Lungen Messe (HHM) ging es auch um Sport - und zwar um \\ den Lungensport zum Zwecke der Rehabilitation und um das Doping zum Zwecke der Leis- \\ tungssteigerung. Bei beiden Themen ist pulmonologischer Sachverstand gefragt.
}

Das HHM-Fortbildungsprogramm wurde gemeinsam von der Klinik für Kardiologie und Angiologie, der Klinik für Herz-, Thorax-, Transplantations- und Gefäßchirurgie (HTTG) und der Klinik für Pneumologie der Medizinischen Hochschule Hannover (MHH) zusammen mit dem Bundesverband Niedergelassener Kardiologen (BNK, Landesverband Niedersachsen) sowie dem Bundesverband Niedergelassener Pneumologen (BNP, Landesverband Niedersachsen) ausgearbeitet.

\section{Sport auch für Patienten mit LVAD}

Lungensport ist eine evidenzbasierte Maßnahme zur Unterstützung der Pharmakotherapie bei Patienten mit chronisch obstruktiven Atemwegs- und Lungenkrankheiten, u. a. bei Asthma, COPD, interstitiellen Lungenkrankheiten, Mukoviszidose und pulmonaler Hypertonie. Sind die gesundheitlichen Voraussetzungen entsprechender Ein- und Ausschlusskriterien gegeben, kann die Teilnahme am Lungensport unter Verwendung des Formulars 56 „Antrag auf Kostenübernahme für Rehabilitationssport" vom Arzt verordnet werden. Das Training besteht aus einem Gruppentraining von mindestens 45 Minuten pro Woche mit höchstens 15 Teilnehmern. Ein qualifizierter Übungs-

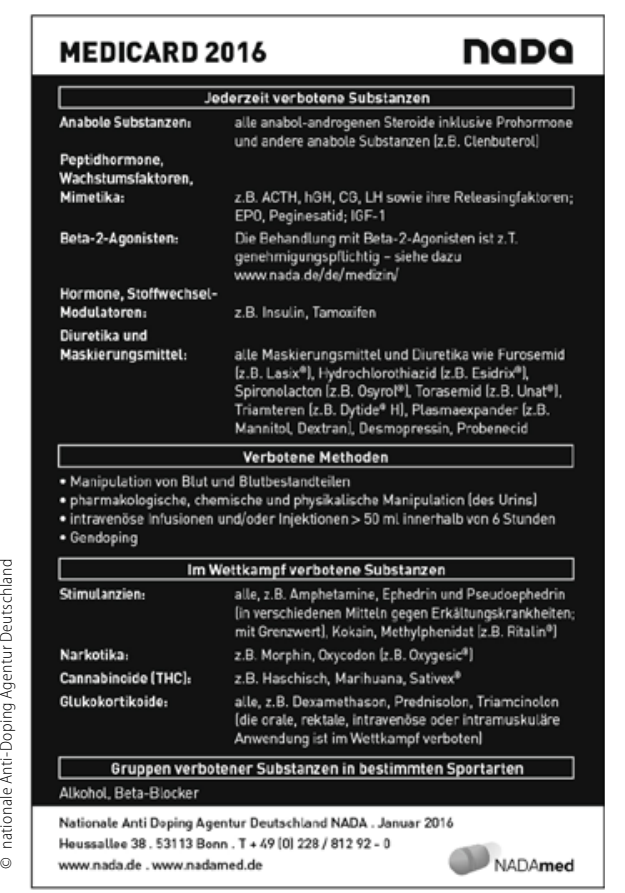

1 Liste verbotener Substanzen beim Sport leiter und ein Arzt in Rufbereitschaft müssen anwesend sein.

Wie Dr. Sven Haufe von der MHH erläuterte, werden beim Lungensport außer Kräftigungs-, Koordinations- und Gleichgewichtsübungen spezielle Atemübungen (Atemtechniken, Sitzpositionen, Lippenbremse) realisiert. Dabei hat sich die $\mathrm{MHH}$ entsprechend ihres herzchirurgischen Schwer- punktes auf den Rehasport bei Patienten mit linksventrikulären Herzunterstützungssystemen (LVAD) spezialisiert. Da immer mehr Patienten ein LVAD nicht nur als Überbrückung bis zur Transplantation, sondern dauerhaft tragen, wurde ein spezielles Heimtrainingsprogramm ausgearbeitet. Es besteht aus einem Ausdauertraining auf einem Smart-Card gesteuerten Ergometer mit Belastungsintensitäten von etwa $10 \%$ unterhalb der Ausdauerleistungsgrenze (etwa 40-50\% der maximalen Sauerstoffaufnahme). Die Patienten trainieren jeden zweiten Tag etwa eine halbe Stunde und machen ergänzend Kräftigungsübungen. Körperliche Aktivität führe zu einer Erhöhung des Pulsdruckes und der Pulsatilität, so Haufe. Ein Pulsdruck-gesteuertes Training könne womöglich dazu beitragen, die Verdünnung der Gefäßwände nach LVAD-Implantation zu reduzieren; dafür geeignete Verfahren müssen aber zunächst noch unter den Bedingungen des Heimtrainings getestet werden.

\section{Doping und Asthma}

Probleme ganz anderer Art haben Leistungssportler, die mithilfe von unerlaubten Substanzen trainieren und Wettkämpfe bestreiten. Sie betreiben Doping, indem sie von den Sportverbänden verbotene Medikamente einnehmen. Die entsprechende Verbotsliste der Welt Anti-Doping Agentur (WADA) wird jedes Jahr aktualisiert und kann in englischer Sprache auf der Homepage der WADA (www.wada-ama.org) eingesehen werden. Für Deutschland gibt es jeweils eine offizielle Übersetzung im Bundesgesetzblatt, außerdem stellt die Nationale Anti-Doping Agentur Deutschland (NADA) die Informationen als sogenannte Medicard ( $\triangle$ Abb. 1) auf ihrer Homepage (www.nada.de) als pdf-Datei zum Download zur Verfügung.

Aus pneumologischer Sicht ist das Verbot vieler Beta-2-Agonisten von Bedeutung. „Ausgenommen sind nur die Inhalation von Salbutamol, Formoterol und Salmeterol, jeweils innerhalb bestimmter Höchstdosierungen“, erläutere Prof. Stephan Sorichter vom St. Josefskrankenhaus in Freiburg. Auch alle oral, intravenös, intramuskulär oder rektal applizierten Glukokortikoide sind für Leistungssportler verboten. Athleten, die eines der erlaubten inhalativen Asthmamittel einnehmen, müssen bei Dopingkontrollen eine entsprechende, von ihrem behandelnden Arzt unterzeichnete Medikamentenmeldung vorlegen. Allen Ärzten, die Leistungssportler betreuen, empfiehlt Sorichter, das jährliche Update der Verbotsliste und die Beispielliste zulässiger Medikamente als kostenloses Download von der NADA zu beziehen.

Dr. Beate Grübler

Quelle: Hannover Herz Lungen Messe, 3.- 5. Juni 2016 